ARTICLE

DOI: $10.1038 /$ ncomms8306 OPEN

\title{
Orbital reconstruction in nonpolar tetravalent transition-metal oxide layers
}

Nikolay A. Bogdanov ${ }^{1}$, Vamshi M. Katukuri ${ }^{1}$, Judit Romhányi ${ }^{1}$, Viktor Yushankhai ${ }^{1,2}$, Vladislav Kataev ${ }^{3}$, Bernd Büchner ${ }^{3,4}$, Jeroen van den Brink ${ }^{1,4}$ \& Liviu Hozoi ${ }^{1}$

A promising route to tailoring the electronic properties of quantum materials and devices rests on the idea of orbital engineering in multilayered oxide heterostructures. Here we show that the interplay of interlayer charge imbalance and ligand distortions provides a knob for tuning the sequence of electronic levels even in intrinsically stacked oxides. We resolve in this regard the $d$-level structure of layered $\mathrm{Sr}_{2} \mid \mathrm{IO}_{4}$ by electron spin resonance. While canonical ligand-field theory predicts $g_{\|}$-factors less than 2 for positive tetragonal distortions as present in $\mathrm{Sr}_{2} \mathrm{IrO}_{4}$, the experiment indicates $g_{\|}$is greater than 2. This implies that the iridium $d$ levels are inverted with respect to their normal ordering. State-of-the-art electronic-structure calculations confirm the level switching in $\mathrm{Sr}_{2} \mathrm{IrO}_{4}$, whereas we find them in $\mathrm{Ba}_{2} \mid \mathrm{lO} \mathrm{O}_{4}$ to be instead normally ordered. Given the nonpolar character of the metal-oxygen layers, our findings highlight the tetravalent transition-metal 214 oxides as ideal platforms to explore $d$-orbital reconstruction in the context of oxide electronics.

\footnotetext{
${ }^{1}$ Institute for Theoretical Solid State Physics, IFW Dresden, Helmholtzstr. 20, 01069 Dresden, Germany. ${ }^{2}$ Joint Institute for Nuclear Research, Joliot-Curie 6 , 141980 Dubna, Russia. ${ }^{3}$ Institute for Solid State Research, IFW Dresden, Helmholtzstr. 20, 01069 Dresden, Germany. ${ }^{4}$ Department of Physics, Technical University Dresden, 01062 Dresden, Germany. Correspondence and requests for materials should be addressed to N.A. B. (email: n.bogdanov@ifw-dresden.de) or to L. H. (email: I.hozoi@ifw-dresden.de).
} 
$\mathrm{T}$ heir unique diversity of transport and magnetic properties endows transition-metal (TM) oxides with a long-term potential for applications in microelectronics and electrical engineering. Nowadays the search for new or superior properties goes beyond known bulk phases and includes oxide interfaces and stacked superlattices ${ }^{1,2}$. As compared with the bulk material, at interfaces the modification of the nearby surroundings can significantly affect the valence electronic structure, in particular, the occupation of the $d$-shell levels ${ }^{1-5}$. This is often referred to as orbital reconstruction ${ }^{3-5}$ and brings to the fore the most basic aspect in electronic-structure theory: how energy levels in quantum matter are formed and populated.

A variety of intrinsically stacked crystalline oxides is presently known. The high-temperature cuprate superconductors ${ }^{6}$, for example, fall in this category but also iridates of the type $\mathrm{A}_{2} \mathrm{IrO}_{4}$ $\left(\mathrm{A}=\mathrm{Sr}^{2+}, \mathrm{Ba}^{2+}\right)$ that closely resemble undoped cuprates, both structurally and magnetically ${ }^{7-11} \cdot \mathrm{Sr}_{2} \mathrm{IrO}_{4}$ has a rather simple crystalline structure displaying stacked, quasi two-dimensional (2D) $\mathrm{IrO}_{2}$ and double $\mathrm{SrO}$ layers. We shall demonstrate that in this system the occupation of the valence $d$ electronic levels differs from what is expected in textbook ligand-field theory due to electrostatics that involves both types of metal-oxygen sheets. In particular, we show that, as compared with the isostructural cuprate $\mathrm{La}_{2} \mathrm{CuO}_{4}$, a different distribution of ionic charges between the TM- $\mathrm{O}_{2}$ and $\mathrm{A}-\mathrm{O}$ layers modifies the sequence of energy levels within the $t_{2 \mathrm{~g}}$ and $e_{\mathrm{g}}$ manifolds and consequently very fundamental physical properties such as the magnetic $g$ factors, which determine the relation between the magnetic moment and quantum number of a magnetic particle. Our findings are of direct relevance to the field of stacked oxide heterostructures and provide a guideline on how low-symmetry crystal fields at $d$-metal sites can be altered and potentially engineered through the appropriate design of successive ionic layers.

To show this we first use electron spin resonance (ESR) measurements to untangle the $5 d$-shell electronic structure of crystalline $\mathrm{Sr}_{2} \mathrm{IrO}_{4}$, in particular, the exact order of the $\operatorname{Ir} t_{2 \mathrm{~g}}$ levels. The single $s=1 / 2$ hole present in these $t_{2 \mathrm{~g}}$ orbitals carries an angular moment $l_{\mathrm{eff}}=1$ and is subject to a large spin-orbit coupling (SOC), which in first approximation results in an effective $\operatorname{Ir}^{4+}$ moment, or pseudospin, $j_{\text {eff }}=l_{\text {eff }}-s \approx 1 / 2$ (refs $7,12,13)$. We compare the experimental properties of these pseudospins with the ones we have calculated by $a b$ initio quantum chemistry methods. This combined approach, explored here on a strongly spin-orbit coupled material for the first time, provides direct access to the spatial anisotropies of the $g$ factors and further to the detailed microscopic superexchange interactions. The ESR measurements and theory are found to agree on a quantitative level and moreover undoubtedly show that the $d$-level ordering in $\mathrm{Sr}_{2} \mathrm{IrO}_{4}$ is inverted with respect to the normal ordering in the sister iridate $\mathrm{Ba}_{2} \mathrm{IrO}_{4}$ or the isostructural 214 cuprate superconductors. The good agreement between the ESR data and the outcome of the computational methodology we describe and employ here establishes the latter as a reliable tool for the investigation of nontrivial electronic structures and magnetic couplings.

\section{Results}

Pseudospins and effective Hamiltonian. Mott-Hubbard physics in $d$-metal compounds has been traditionally associated with first-series (3d) TM oxides. However, recently, one more ingredient entered the TM-oxide 'Mottness' paradigm-large SOC's in $5 d$ systems. SOC in $5 d$ and to some extent $4 d$ anisotropic oxides modifies the very nature of the correlation hole of an electron, by admixing the different $t_{2 \mathrm{~g}}$ components ${ }^{12,13}$, changes the conditions for localization ${ }^{7}$, the criteria of Mottness and further gives rise to new types of magnetic ground states and excitations ${ }^{9,11}$. While various measurements indicate that indeed spin-orbit-coupled $j_{\text {eff }} \approx 1 / 2$ states form in $\mathrm{A}_{2} \mathrm{IrO}_{4}$ (refs 7,9,14), it has been also pointed out that off-diagonal SOC's may mix into the ground state (GS) wavefunction substantial amounts of $t_{2 \mathrm{~g}}^{4} e_{\mathrm{g}}^{1}$ character ${ }^{15-18}$. Such $t_{2 \mathrm{~g}}^{5}-t_{2 \mathrm{~g}}^{4} e_{\mathrm{g}}^{1}$ many-body interactions were shown to produce remarkable effects in X-ray absorption and X-ray magnetic circular dichroism (XMCD): the branching ratio between the $L_{3}$ and $L_{2} \operatorname{Ir} 2 p$ absorption edges reaches values as large as 4 , nearly $50 \%$ higher than the 2.75 value for a 'pure' $j_{\text {eff }}=1 / 2$ system $^{19}$. In addition, low-symmetry noncubic fields produce sizeable splittings of the $5 d t_{2 \mathrm{~g}}$ levels, in some cases close to or even larger than $\sim 1 / 2 \mathrm{eV}$ (refs 20-22), and therefore admix the $j_{\text {eff }}=1 / 2$ and $j_{\text {eff }}=3 / 2 t_{2 \mathrm{~g}}^{5}$ components ${ }^{13,15}$. The structure of the spin-orbit GS depends on both the strength and sign of these splittings. Interestingly, the best fits of the X-ray absorption and XMCD data are achieved in $\mathrm{Sr}_{2} \mathrm{IrO}_{4}$ with a negative $t_{2 \mathrm{~g}}$ tetragonal splitting ${ }^{19}$, although the oxygen octahedra in this material display a distinct positive tetragonal distortion-the $\mathrm{IrO}_{6}$ octahedra are substantially elongated $^{23}$ (a negative tetragonal splitting should occur when the $\mathrm{IrO}_{6}$ octahedra are compressed ${ }^{13,24}$, see Fig. 1). This is already a first indication of the level inversion that our ESR measurements and quantum chemistry calculations show to take place in $\mathrm{Sr}_{2} \mathrm{IrO}_{4}$.

The interactions between a pair $\langle i j\rangle$ of nearest-neighbour (NN) $1 / 2$ pseudospins in the presence of an external magnetic field $\mathbf{h}$ is given by the effective Hamiltonian

$$
\mathcal{H}_{\mathrm{eff}}^{i, j}=J \tilde{\mathbf{S}}_{i} \cdot \tilde{\mathbf{S}}_{j}+\mathbf{D} \cdot \tilde{\mathbf{S}}_{i} \times \tilde{\mathbf{S}}_{j}+\tilde{\mathbf{S}}_{i} \cdot \overline{\bar{\Gamma}} \cdot \tilde{\mathbf{S}}_{j}+\mu_{B} \sum_{k=i, j} \mathbf{h} \cdot \overline{\mathbf{g}}_{k} \cdot \tilde{\mathbf{S}}_{k},
$$

where $\tilde{\mathbf{S}}_{i}, \tilde{\mathbf{S}}_{j}$ are pseudospin $\left(j_{\text {eff }} \approx 1 / 2\right)$ operators, $J$ is the isotropic Heisenberg exchange, $\mathbf{D}=(0,0, D)$ defines the antisymmetric Dzyaloshinskii-Moriya (DM) coupling, $\overline{\bar{\Gamma}}$ is a symmetric traceless second-rank tensor describing the symmetric anisotropy and due to the staggered rotation of the $\mathrm{IrO}_{6}$ octahedra the $\overline{\overline{\mathbf{g}}}$ tensor splits for each of the two sites into uniform and staggered components $\overline{\overline{\mathbf{g}}}_{k}^{\mathrm{u}} \pm \overline{\overline{\mathbf{g}}}_{k}^{s}$ (see for example, refs 16,25). This effective spin Hamiltonian is of direct relevance to the interpretation of the ESR data.

ESR measurements. For a single crystal of $\mathrm{Sr}_{2} \mathrm{IrO}_{4}$ we observe antiferromagnetic resonance (AFR) modes in the sub-THz frequency domain ${ }^{26}$ as displayed in Fig. 2. There are two modes
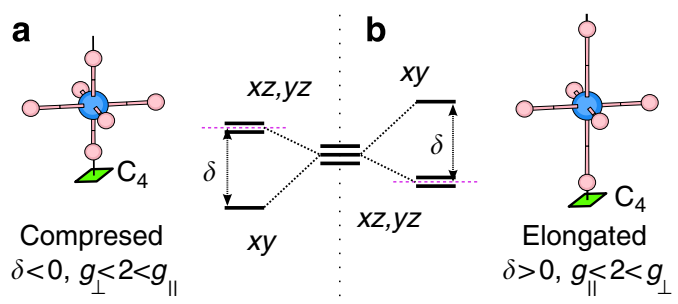

Figure $1 \mid \mathrm{TM} \boldsymbol{t}_{2 \mathrm{~g}}$ splittings for tetragonal distortions of the oxygen octahedron sans SOC. (a) $z$ axis compression of the octahedron corresponds to a tetragonal splitting $\delta<0$, causes an orbital dublet to be lowest in energy and the $g$ factors to be ordered as $g_{\perp}<2<g_{\|}$.

(b) Elongation of the octahedron $(\delta>0)$ causes an orbital singlet to be lowest in energy and the $g$ factors to be ordered as $g_{\|}<2<g_{\perp}$. Purple dashed lines indicate the conventional zero level used to define the sign of $\delta$. 

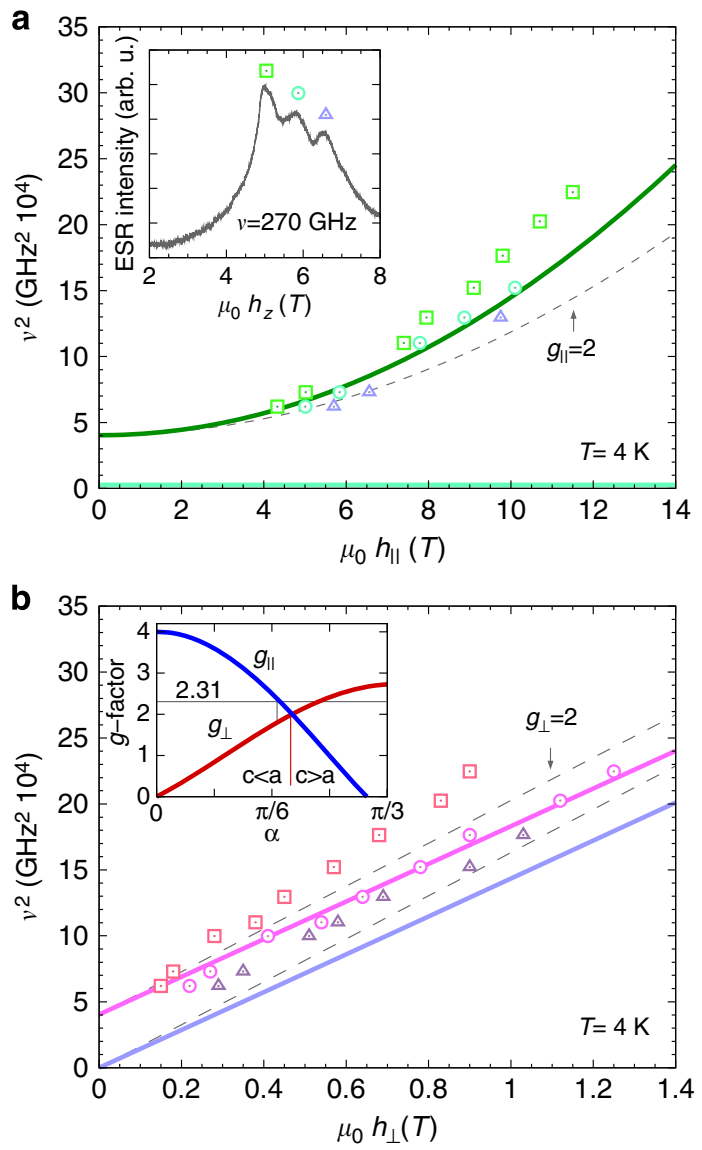

Figure 2 | ESR data for $\mathbf{S r}_{2} \mathrm{IrO}_{\mathbf{4}}$. (a) Out-of-plane magnetic-field geometries; the inset shows a representative AFR spectrum. (b) In-plane magnetic fields; the inset demonstrates the $g$-factor anisotropy as function of the tetragonal distortion parameter $\alpha$ (see text). Symbols denote experimental data points - solid lines are theoretical curves using equations $(2,3,4)$ and the quantum chemically computed $g$ factors $g_{\|}=2.31, g_{\perp}=1.76$ (see Table 1); dashed lines are calculated assuming isotropic $g$ factors.

if $\mathbf{h} \| z$ : a gapless Goldstone mode $v_{\| 1}=0$ and a gapped excitation

$$
v_{\| 2}^{2}=\Delta^{2}+2 g_{\|}^{2} h_{\|}^{2} \tilde{J} /\left(2\left(J+\Gamma_{z z}\right)+\tilde{J}\right)
$$

where $\Delta=\sqrt{2 \tilde{J}\left(\tilde{J}-2\left(J+\Gamma_{z z}\right)\right)}, \quad \tilde{J}=\sqrt{4 D^{2}+\left(2 J-\Gamma_{z z}\right)^{2}}$ and $\Gamma_{z z}$ couples the $\tilde{S}_{i}^{z}$ and $\tilde{S}_{j}^{z}$ components (along the $c$ axis, perpendicular to the $a b$-plane $\mathrm{IrO}_{2}$ layers ${ }^{23}$ ) in the third term of equation (1) (see Methods for details). Experimental results are shown for $v_{\| 2}$ in Fig. 2a. The data comprise at $T \ll T_{\mathrm{N}}=240 \mathrm{~K}$ a group of overlapping resonances (Fig. 2a, inset), possibly due to some distribution of internal fields in the sample. Though revealing some scatter, the $v_{\| 2}^{2}$ AFR data follow approximately a parabolic dependence on $h$ and, most importantly, they lie substantially above the curve corresponding to the free-electron Landé factor $g_{\mathrm{e}}=2$ (dashed line in Fig. 2a). The experimental dependence $v_{\| 2}^{2}\left(h^{2}\right)$ can be reasonably well modelled with $g_{\|}$values of 2.3-2.45. The solid line in Fig. 2a is obtained by using $g_{\|}=2.31$, as derived from quantum chemistry calculations that will be discussed later on.

Sizeable deviations to values $>2$ of $g_{\|}$is clear indication of the presence of low-symmetry, noncubic crystal fields. In the simplest approximation, that is, restricting ourselves to the $\mathrm{Ir}^{4+}$ $t_{2 \mathrm{~g}}^{5}$ manifold, the anisotropic $g$ factors in axial noncubic environment can be expressed up to the sign as ${ }^{13} g_{\|}=g_{\mathrm{c}}=$ $(2+2 k) \cos ^{2} \alpha-2 \sin ^{2} \alpha$ and $g_{\perp}=g_{a}=g_{b}=2 \sin ^{2} \alpha+2 \sqrt{2} k \cos \alpha \sin \alpha$, where $k$ is a covalency reduction factor, $\alpha=(1 / 2)$ arctan $[2 \sqrt{2} \lambda /(\lambda-2 \delta)]$ parameterizes the deviation from octahedral symmetry, $\lambda$ is the SOC constant and $\delta$ the $\operatorname{Ir} t_{2 \mathrm{~g}}$ splitting. A plot for the dependence of the diagonal $g$ factors on the distortion parameter $\alpha$ is shown in the inset to Fig. 2b. For simplicity, $k=1$ is for the moment assumed but smaller values of $k$ do not bring qualitative changes. In cubic symmetry $\delta=0, \alpha_{\mathrm{cub}}=35.26^{\circ}$ and the $g$ matrix is isotropic with $g_{\|}=g_{\perp}=2$. According to standard textbooks on ligand-field theory ${ }^{24}$, an elongation of the out-ofplane Ir-O bond induces a positive tetragonal splitting of the Ir $t_{2 g}$ levels, with $\delta>0$ and $\alpha>\alpha_{\text {cub }}$, whereas a bond compression yields $\delta<0$ and $\alpha<\alpha_{\text {cub }}$ (see Fig. 1). As in $\mathrm{Sr}_{2} \mathrm{IrO}_{4}$ the $\mathrm{IrO}_{6}$ octahedra are substantially elongated in the $z$ direction ${ }^{23}$, the $g$ factors are expected to correspond to the case of positive splitting $\alpha>\alpha_{\mathrm{cub}}$, see the area to the right of the crossing point shown in the inset to Fig. 2b. It follows from the plot that $g_{\|}<2$, which obviously contradicts our AFR data for $\mathbf{h} \| z$ [Fig. 2a]. The value $g_{\|}=2.31$ used to draw the curve connecting the open circles in Fig. $2 \mathrm{a}$ in fact corresponds to $\alpha=32.05^{\circ}<\alpha_{\text {cub }}$ (see the inset in Fig. 2b) and indicates that, despite the positive $c$-axis tetragonal distortion, a counterintuitive negative tetragonal splitting of the $5 d t_{2 \mathrm{~g}}$ levels is present in $\mathrm{Sr}_{2} \mathrm{IrO}_{4}$.

It should be noted that there is no Goldstone mode for finite in-plane magnetic fields. The canting angle depends in this case both on the strength of the DM interaction and the applied field. The two modes are

$$
v_{\perp 1}^{2}=m^{2} g_{\perp}^{2} h_{\perp}^{2}+m g_{\perp} h_{\perp}\left(-4 J-\Gamma_{z z}+m J^{\prime}\right)
$$

and

$$
\begin{aligned}
v_{\perp 2}^{2} & =m^{2} g_{\perp}^{2} h_{\perp}^{2}+m g_{\perp} h_{\perp}\left(-4 J+5 \Gamma_{z z}+3 m J^{\prime}\right) \\
& +\left(-4 J+2 \Gamma_{z z}+2 m J^{\prime}\right)\left(3 \Gamma_{z z}+m J^{\prime}\right),
\end{aligned}
$$

where $J^{\prime}=-4 D \sqrt{1-m^{2}}+2 m\left(2 J-\Gamma_{z z}\right)$ and $m$ is the in-plane ferromagnetic component of the effective moments. To first order in magnetic field, $m$ can be expressed as

$$
m=-\sqrt{\frac{\tilde{J}-2 J+\Gamma_{z z}}{2 \tilde{J}}}-\frac{g_{\perp} h_{\perp}}{2 \sqrt{6 D^{2}+\left(2 J-\Gamma_{z z}\right)^{2}}},
$$

which holds for weak fields where $m$ remains small. The first term corresponds to the zero-field canting, arising from the DM interaction, while the second term shows how this canting evolves with increasing $h_{\perp}$. Plots based on equations $(3,4)$ and the quantum chemically derived interaction parameters (see below) are displayed in Fig. $2 \mathrm{~b}$ together with ESR data.

Quantum chemistry calculations of $\boldsymbol{g}$ factors. Results of $a b$ initio quantum chemistry calculations for the $g$ factors in $\mathrm{Sr}_{2} \mathrm{IrO}_{4}$ and in the structurally related material $\mathrm{Ba}_{2} \mathrm{IrO}_{4}$ are listed in Table 1 . Our computational scheme follows the prescription of Bolvin ${ }^{27}$ and Vancoillie et al. ${ }^{28}$. It maps the matrix elements (MEs) of the ab initio Zeeman Hamiltonian $\mathcal{H}_{\mathrm{Z}}=-\boldsymbol{\mu} \cdot \mathbf{h}=\mu_{\mathrm{B}}\left(\mathbf{L}+g_{\mathrm{e}} \mathbf{S}\right) \cdot \mathbf{h}$ onto the MEs of the effective pseudospin Hamiltonian $\mathcal{H}_{\mathrm{S}}=\mu_{\mathrm{B}} \mathbf{h} \cdot \overline{\mathbf{g}} \cdot \tilde{\mathbf{S}}$, where $\boldsymbol{\mu}, \mathbf{L}$ and $\mathbf{S}$ are magnetic moment, angular-momentum and spin operators, respectively. The spinorbit GS wave functions are computed either at the completeactive-space self-consistent-field (CASSCF) or multireference configuration-interaction (MRCI) level of theory ${ }^{29}$, as described in ref. 30 and using the MOLPRO quantum chemistry package ${ }^{31}$. All necessary angular-momentum MEs are calculated as well with MOLPRO (see Methods). In a first set of calculations, only the three $t_{2 \mathrm{~g}}$ orbitals at a given Ir site and five electrons were considered in the active space. The self-consistent-field 
Table 1 | $g$ factors for $\mathrm{Sr}_{2} \mathrm{IrO}_{4}$ and $\mathrm{Ba}_{2} \mathrm{IrO}_{4}$.

\begin{tabular}{|c|c|c|c|c|}
\hline \multirow[t]{2}{*}{ States considered } & \multicolumn{2}{|c|}{ CASSCF } & \multicolumn{2}{|c|}{ MRCI } \\
\hline & $\boldsymbol{g}_{\perp}$ & $g_{\|}$ & $g_{\perp}$ & $g_{\|}$ \\
\hline \multicolumn{5}{|l|}{$\mathrm{Sr}_{2} \mathrm{IrO}_{4}(\delta=-155 \mathrm{meV}):$} \\
\hline$t_{2 g}^{5}(3 \mathrm{KDs})$ & 1.67 & 2.25 & 1.60 & 2.35 \\
\hline \multicolumn{5}{|l|}{$\mathrm{Ba}_{2} \mid \mathrm{IOO}_{4}(\delta=65 \mathrm{meV}):$} \\
\hline$t_{2 g}^{5}(3 \mathrm{KDs})$ & 2.00 & 1.61 & 2.01 & 1.60 \\
\hline
\end{tabular}

CASSCF = complete-active-space self-consistent-field; $\mathrm{KDs}=$ Kramers doublets; $\mathrm{MRCl}=$ multireference configuration-interaction. Results of many-body quantum chemistry calculations are shown. The left column displays the electron configurations entering the spin-orbit treatment. Only the high-spin sextet state is considered out of the $t_{2 g}^{3} e_{g}^{2}$ manifold. Final results are indicated in bold face.

optimization was carried out for the corresponding ${ }^{2} T_{2 \mathrm{~g}}\left(t_{2 \mathrm{~g}}^{5}\right)$ state. We use here the more convenient notations associated to $\mathrm{O}_{h}$ symmetry, although the calculations were performed for the actual experimental geometry, with point-group symmetry lower than octahedral. Inclusion of SOC yields in this case a set of three Kramers doublets (KDs), see Table 1.

Subsequently we performed calculations with larger active spaces, including also the Ir $e_{\mathrm{g}}$ orbitals. One $t_{2 \mathrm{~g}}^{5}\left({ }^{2} T_{2 \mathrm{~g}}\right)$ plus four $t_{2 \mathrm{~g}}^{4} e_{\mathrm{g}}^{1}\left({ }^{2} A_{2 \mathrm{~g}},{ }^{2} T_{1 \mathrm{~g}},{ }^{2} E_{\mathrm{g}}\right.$ and $\left.{ }^{2} T_{2 \mathrm{~g}}\right)$ spin doublets, two spin quartets $\left[{ }^{4} T_{1 \mathrm{~g}}\left(t_{2 \mathrm{~g}}^{4} e_{\mathrm{g}}^{1}\right)\right.$ and $\left.{ }^{4} T_{2 \mathrm{~g}}\left(t_{2 \mathrm{~g}}^{4} e_{\mathrm{g}}^{1}\right)\right]$ and one spin sextet $\left[{ }^{6} A_{1 \mathrm{~g}}\left(t_{2 \mathrm{~g}}^{3} e_{\mathrm{g}}^{2}\right)\right]$ entered here the spin-orbit treatment. The orbitals were optimized for an average of all these terms.

The effect of enlarging the active space to include $t_{2 \mathrm{~g}}^{4} e_{\mathrm{g}}^{1}$ terms in the reference wavefunction is in the range of $10 \%$, in line with earlier semi-empirical estimates for $4 d^{5}$ and $5 d^{5}$ systems ${ }^{15-18}$. Most importantly, the calculations yield a negative tetragonal splitting of the Ir $t_{2 \mathrm{~g}}$ levels in $\mathrm{Sr}_{2} \mathrm{IrO}_{4}, \delta=-155 \mathrm{meV}$ by MRCI, and positive $t_{2 \mathrm{~g}}$ splitting in $\mathrm{Ba}_{2} \mathrm{IrO}_{4}$ (see Table 1 and Methods). Similar signs, negative in $\mathrm{Sr}_{2} \mathrm{IrO}_{4}$ and positive in $\mathrm{Ba}_{2} \mathrm{IrO}_{4}$, but much larger magnitudes $(\approx 0.7 \mathrm{eV})$ are found for the computed Ir $e_{\mathrm{g}}$ splittings (not shown in Table 1).

Taken together, the ESR and quantum chemistry results unequivocally point at an anomalous order of the split Ir $5 d$ levels in $\mathrm{Sr}_{2} \mathrm{IrO}_{4}$, related to the important role of the extended crystalline surroundings in generating low-symmetry fields that compete with 'local' distortions of the ligand cage. Similar effects were found by $a b$ initio calculations on the 214 layered rhodate $\mathrm{Sr}_{2} \mathrm{RhO}_{4}$ (ref. 32) and the 227 pyrochlore iridates ${ }^{22}$. In contrast, in $\mathrm{Ba}_{2} \mathrm{IrO}_{4}$, the stretch of the apical Ir-O bonds is strong enough ${ }^{33}$ to overcome the longer-range electrostatics, turning the tetragonal $t_{2 \mathrm{~g}}$ positive again, as discussed in more detail in the following. Consequently, the structure of the $\overline{\overline{\mathbf{g}}}$ tensor in $\mathrm{Ba}_{2} \mathrm{IrO}_{4}$ is qualitatively different, with $g_{\perp}>2$ and $g_{\|}<2$ (see Table 1), the ordering that one normally expects and encounters for elongated octahedra $^{13}$.

Exchange couplings from quantum chemistry. To obtain $a b$ initio quantum chemistry values for the inter-site effective magnetic couplings in $\mathrm{Sr}_{2} \mathrm{IrO}_{4}$ (see equation (1)), we carried out additional calculations on larger clusters that incorporate two $5 d^{5}$ sites. The two-octahedra cluster has $C_{2 \mathrm{v}}$ symmetry (see Fig. 3 ), which implies a diagonal form for $\overline{\bar{\Gamma}}$ and $\overline{\mathbf{g}}_{i}^{\mathrm{u}}$ in equation (1) (see Methods). By one-to-one correspondence between the MEs of the $a b$ initio Hamiltonian

$$
\mathcal{H}^{i, j}=\mathcal{H}_{\mathrm{SR}}^{i, j}+\mathcal{H}_{\mathrm{SO}}^{i, j}+\mathcal{H}_{\mathrm{Z}}^{i, j}
$$

and the MEs of the effective spin Hamiltonian (1) in the basis of the lowest four spin-orbit states defining the magnetic spectrum of two NN octahedra, we can derive in addition to the $g$ factors the

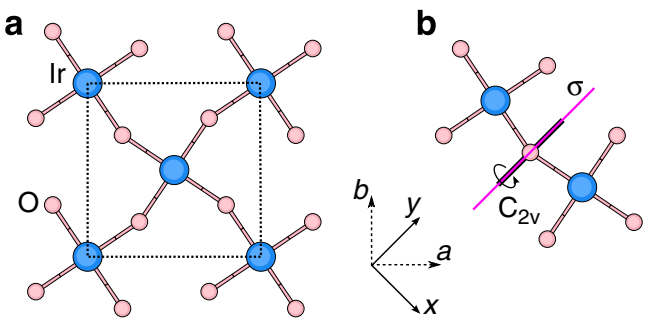

Figure 3 | Planar $\mathbf{I r O}_{\mathbf{2}}$ network in $\mathbf{S r}_{\mathbf{2}} \mathbf{I r O} \mathbf{O}_{\mathbf{4}}$ (a) Coordination of the Ir site. Dashed lines show the boundaries of the crystallographic unit cell within a given $\mathrm{IrO}_{2}$ layer. (b) The point-group symmetry of the $\left[\mathrm{Ir}_{2} \mathrm{O}_{11}\right]$ block is $\mathrm{C}_{2 \mathrm{v} \text { i }}$; associated symmetry elements are indicated in the figure.

Table 2 | Nearest-neighbour magnetic couplings in $\mathrm{Sr}_{2} \mathrm{IrO}_{4}$

\begin{tabular}{lcccc}
$\boldsymbol{J}$ & $\boldsymbol{D}$ & $\boldsymbol{\Gamma}_{\mathbf{x x}}$ & $\boldsymbol{\Gamma}_{\mathbf{y} \mathbf{y}}$ & $\boldsymbol{\Gamma}_{\mathbf{z z}}$ \\
\hline 47.8 & \pm 11.9 & 0.42 & -0.84 & 0.42
\end{tabular}

Results of spin-orbit MRCl calculations on two-octahedra clusters are displayed (meV). $\Gamma_{x x}+\Gamma_{y y}+\Gamma_{z z}=0$ since $\overline{\bar{\Gamma}}$ is traceless.

strengths of the Heisenberg and anisotropic intersite couplings. In equation (6), $\mathcal{H}_{\mathrm{SR}}^{i, j}$ is the scalar-relativistic BornOppenheimer Hamiltonian, $\mathcal{H}_{\mathrm{SO}}^{i, j}$ describes spin-orbit interactions ${ }^{30}$ and $\mathcal{H}_{\mathrm{Z}}^{i, j}$ is the two-site Zeeman Hamiltonian.

Diagonalization of the spin Hamiltonian (1) provides the expected singlet $|\tilde{s}\rangle$ and three (split) triplet components $\left|t_{x}\right\rangle,\left|t_{y}\right\rangle$ and $\left|\tilde{t}_{z}\right\rangle$. Owing to the DM interaction, $|\tilde{s}\rangle$ and $\left|\tilde{t}_{z}\right\rangle$ are admixtures of 'pure' $|0,0\rangle$ and $|1,0\rangle$ spin functions. Our mapping procedure yields $J \approx 48 \mathrm{meV}$, somewhat lower than $J$ values of $55-60 \mathrm{meV}$ derived from experiment ${ }^{9,34}$, and a ratio between the DM and Heisenberg couplings $D / J=0.25$, in agreement with estimates based on effective superexchange models ${ }^{12,35,36}$ and large enough to explain the nearly rigid rotation of magnetic moments that is observed when the $\mathrm{IrO}_{6}$ octahedra revolve ${ }^{37,38}$. Ab initio results for the NN anisotropic couplings $\overline{\bar{\Gamma}}$, also relevant for a detailed understanding of the magnetic properties of $\mathrm{Sr}_{2} \mathrm{IrO}_{4}$, are shown as well in Table 2 . In our convention the $x$ axis is taken along the Ir-Ir link, that is, it coincides with the $\langle 110\rangle$ crystallographic direction $^{23}$, and $z \| c$. We obtain $\Gamma_{x x} \approx \Gamma_{z z}$, which then allows to recast the Heisenberg and symmetric anisotropic terms in (1) as $\left(J-\Gamma_{y y} / 2\right) \tilde{\mathbf{S}}_{i} \cdot \tilde{\mathbf{S}}_{j}+\left(3 \Gamma_{y y} / 2\right) \tilde{S}_{i}^{y} \tilde{S}_{j}^{y}, \quad$ with $\Gamma_{x x}=\Gamma_{z z}=-\Gamma_{y y} / 2$. Equally interesting, for no rotation of the $\mathrm{IrO}_{6}$ octahedra and straight Ir-O-Ir bonds in $\mathrm{Ba}_{2} \mathrm{IrO}_{4}$, it is $\Gamma_{y y}$ and $\Gamma_{z z}$ which are 
approximately the same, providing a realization of the compassHeisenberg model ${ }^{36,39}$ since the DM coupling is by symmetry 0 in that case.

The two-site magnetic Hamiltonian (1) features in-plane symmetric-anisotropy couplings $\Gamma_{x x}$ and $\Gamma_{y y}$, which were not considered in previous studies ${ }^{12,26}$. In the presence of twosublattice order, terms containing these couplings cancel each other in the mean-field energy but they are in general relevant for pseudospin fluctuations and excitations. Using spin-wave theory and effective parameters derived from the quantum chemistry calculations, we nicely reproduce the correct GS and character of the modes, as shown in Fig. 2. To reproduce the experimental zero-field gap, in particular, we used $J, D$ and $g$-factor values as listed in Table 2 and a somewhat larger $\Gamma_{z z}$ parameter of $0.98 \mathrm{meV}$. To leading order, the dependence of $v_{\perp 1}^{2}$ and $v_{\perp 2}^{2}$ on $h$ is linear, see equations $(3,4)$, and the slope is proportional to $m$. At low fields $(\leq 1 \mathrm{~T}), m$ can be actually replaced with its field independent value ${ }^{26}$. Using the MRCI coupling constants, the first term in equation (5) then yields a moment $m \approx 0.12 \mu_{\mathrm{B}}$, in good agreement with the experiment ${ }^{8,40}$.

\section{Discussion}

The exact $d$-level order is of fundamental importance in TM oxides, dictating for instance the symmetry of the quasiparticle states in photoemission ${ }^{10,32,41}$ and the nature of the magnetic ordering $^{42,43}$. In 214 iridates specifically, it determines the various isotropic as well as anisotropic contributions to the magnetic exchange couplings ${ }^{12,35,36,39}$, the evolution of those magnetic interactions with strain ${ }^{44}$ and/or pressure ${ }^{19}$ and most likely the nature of the intriguing transition to a nonmagnetic phase in $\mathrm{Sr}_{2} \mathrm{IrO}_{4}$ under high pressure ${ }^{19}$. Having established that in $\mathrm{Sr}_{2} \mathrm{IrO}_{4}$ the $d$ levels are inverted and that in the closely related $\mathrm{Ba}_{2} \mathrm{IrO}_{4}$ they are not raises the question what actually drives the inversion. To address this, we performed an additional set of calculations, in which we change the charges around the reference $\mathrm{IrO}_{6}$ octahedron. As a simple numerical experiment that preserves charge neutrality of the $\mathrm{A}_{2} \mathrm{IrO}_{4}$ system, we assigned the $4 \mathrm{NN}$ iridium sites (in-plane, see Fig. 4) the charge $Q_{\mathrm{TM}}-2 \Delta q$ and the 8 closest $\mathrm{A}$-site cations (out of plane) the valence $Q_{\mathrm{A}}+\Delta q$. In a fully ionic picture, $Q_{\mathrm{TM}}$ and $Q_{\mathrm{A}}$ are $4+$ and $2+$, respectively. However, since in our calculations the NN TM and A sites are not modelled as just formal point charges (see Methods), the actual valence states depart from their formal values, with larger 'deviations' for $Q_{\mathrm{TM}}$. The way we introduce $\Delta q$ in the
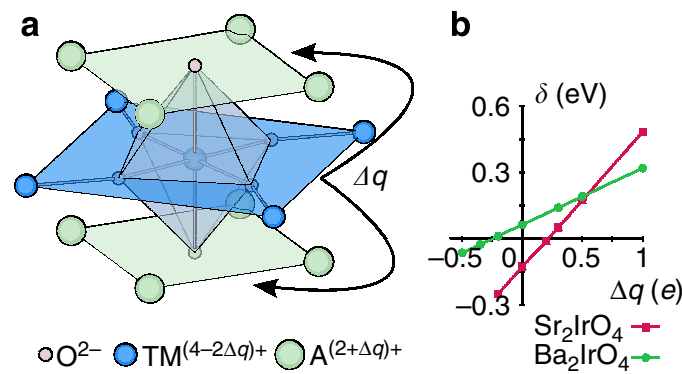

Figure 4 | Effect of interlayer charge imbalance in $\mathbf{A}_{\mathbf{2}} \mathbf{I r O} \mathbf{O}_{\mathbf{4}}$ iridates.

(a) The nearby surroundings of $\mathrm{TM}$ sites in $\mathrm{A}_{2} \mathrm{TMO}_{4}$-layered perovskites. In test calculations one can assign the adjacent (in-plane) TM ions the formal charge $\mathrm{Q}_{\mathrm{TM}}-2 \Delta q$, which is compensated by assigning the NN A sites the charge $\mathrm{Q}_{\mathrm{A}}+\Delta q$. (b) Tetragonal crystal-field energy splitting between $t_{2 \mathrm{~g}}$ orbitals $(\delta)$ as a function of the charge redistribution $\Delta q$ for $\mathrm{Sr}_{2} \mathrm{IrO}_{4}$ and $\mathrm{Ba}_{2} \mathrm{IrO}_{4}$. computations is therefore by appropriately modifying the nuclear charge at the respective site. For variable $\Delta q$, this interpolates linearly between nearby surroundings corresponding to $5 d 214$ layered perovskites (with $\Delta q=0$ and $\mathrm{TM}^{4+}, \mathrm{A}^{2+}$ formal valence states) and their cuprate 214 equivalents (with $\Delta q=1$, $\mathrm{TM}^{2+} / \mathrm{A}^{3+}$ formal ionic charges and 'normal' order of the TM $t_{2 \mathrm{~g}}$ and $e_{\mathrm{g}}$ levels ${ }^{45}$ ).

As is illustrated in Fig. $4 \mathrm{a}$, increasing $\Delta q$ amounts to moving positive charge from the $\mathrm{IrO}_{2}$ plane to the adjacent $\mathrm{A}-\mathrm{O}$ layers. The calculations show that upon moving charge in such a manner, the Ir $t_{2 \mathrm{~g}}$ splitting $\delta$ increases, see Fig. $4 \mathrm{~b}$. In other words, this redistribution of charge counteracts the level inversion in $\mathrm{Sr}_{2} \mathrm{IrO}_{4}$ and further increases the already positive $\delta$ in $\mathrm{Ba}_{2} \mathrm{IrO}_{4}$. In $\mathrm{Sr}_{2} \mathrm{IrO}_{4}$ the cubic-like $j_{\text {eff }}=1 / 2$ limit occurs for $\Delta q=0.22$. This effect can easily be understood: placing more positive charge out of the $\mathrm{IrO}_{2}$ plane stabilizes the out-of-plane $t_{2 \mathrm{~g}}$ orbitals, corresponding to the $(y z, z x)$ orbital doublet, and thus enhances $\delta$. One can also do the opposite and drive $\Delta q$ negative. In this case more positive charge piles up in the $\mathrm{IrO}_{2}$ plane, which one expects to lower the energy of the $x y$ orbital singlet, thus enhancing the level inversion in $\mathrm{Sr}_{2} \mathrm{IrO}_{4}$. This is indeed what happens, see Fig. $4 \mathrm{~b}$. What is more, driving $\Delta q$ negative even causes a level inversion in $\mathrm{Ba}_{2} \mathrm{IrO}_{4}$, when $\Delta q \lesssim-0.25$. It is interesting to note that the slope of the $\delta$ versus $\Delta q$ lines in $\mathrm{Sr}_{2} \mathrm{IrO}_{4}$ is much steeper than in $\mathrm{Ba}_{2} \mathrm{IrO}_{4}$, which is caused by the significantly smaller Ir-Ir distances in $\mathrm{Sr}_{2} \mathrm{IrO}_{4}$.

From these test calculations it is clear that low-symmetry crystal fields associated to neighbours beyond the first ligand coordination shell, in particular, the highly charged $\mathrm{Ir}^{4+} \mathrm{NNs}$, counteract the local tetragonal crystal field that is caused by the elongation of the $\mathrm{IrO}_{6}$ octahedra in both $\mathrm{Sr}_{2} \mathrm{IrO}_{4}$ and $\mathrm{Ba}_{2} \mathrm{IrO}_{4}$. In the case of $\mathrm{Ba}_{2} \mathrm{IrO}_{4}$ the local distortion is still strong enough to overcome these longer-range effects but in $\mathrm{Sr}_{2} \mathrm{IrO}_{4}$, with a slightly smaller tetragonal distortion, the longer-range electrostatics wins, causing the observed level inversion.

While the role of the high ionic charge of in-plane ions has been earlier invoked in the tetravalent $\mathrm{Ru}$ oxide compound $\mathrm{Ca}_{2} \mathrm{RuO}_{4}$ (ref. 43) and in mixed-valence manganites ${ }^{46}$, we here explicitly prove it by combined ESR measurements and many-body $a b$ initio calculations on structurally and chemically simpler systems in which additional complications arising from further distortions ${ }^{43,47}$ or the presence of multiple TM valence states $^{46}$ are excluded. A reversed order of the Ir $t_{2 g}$ levels in $\mathrm{Sr}_{2} \mathrm{IrO}_{4}$ has been also indirectly implied by fits of X-ray absorption $^{19}$ and X-ray magnetic scattering ${ }^{37,48}$ spectra. As a more direct and more sensitive experimental technique to such details of the valence electronic structure and with back up from truly $a b$ initio many-body calculations, ESR now provides irrefutable evidence for such physics. The numerical 'experiment' outlined in Fig. 4 further shows that at the heart of this effect is not the intersite exchange, as assumed in ref. 19, and not the $t_{2 \mathrm{~g}}-e_{\mathrm{g}}$ orbital hybridization invoked in ref. 48 , but basic interlayer electrostatics.

We have, in sum, provided an integrated picture on the $d$-level structure and magnetic anisotropies in $\mathrm{Sr}_{2} \mathrm{IrO}_{4}$, a prototype spinorbit driven magnetic insulator. Both the single-site $\overline{\overline{\mathbf{g}}}$ tensor and intersite effective exchange interactions are analysed in detail. To access the latter, we build on an earlier computational scheme for deriving intersite matrix elements in mixed-valence spin-orbit coupled systems ${ }^{49}$. While the ratio $D / J$ of the antisymmetric Dzyaloshinskii-Moriya and isotropic Heisenberg couplings is remarkably large in $\mathrm{Sr}_{2} \mathrm{IrO}_{4}$ and concurs with an in-plane rotation pattern of the Ir magnetic moments that follows nearly rigidly the staggered rotation of the $\mathrm{IrO}_{6}$ octahedra ${ }^{37,38}$, the most prominent symmetric anisotropic terms are according to the quantum chemistry data in-plane, perpendicular to the Ir-Ir links. 
The structure of the $\overline{\overline{\mathbf{g}}}$ tensor, as measured by ESR and computed with first-principles electronic-structure methods, is such that $g_{\|}=g_{c}>g_{\perp}=g_{a}=g_{b}$ and distinctly indicates a negative tetragonal-like splitting of the $\operatorname{Ir} t_{2 \mathrm{~g}}$ levels, in spite of sizable positive tetragonal distortions in $\mathrm{Sr}_{2} \mathrm{IrO}_{4}$. We further observe that a much stronger tetragonal distortion in $\mathrm{Ba}_{2} \mathrm{IrO}_{4}$ renders the tetragonal $d$-level splitting positive and $g_{\|}<g_{\perp}$. The interesting situation arises that nevertheless the magnitude of the $\operatorname{Ir} t_{2 g}$ splitting is largest in $\mathrm{Sr}_{2} \mathrm{IrO}_{4}$. The $d$-level inversion in $\mathrm{Sr}_{2} \mathrm{IrO}_{4}$ and the surprisingly small splitting in $\mathrm{Ba}_{2} \mathrm{IrO}_{4}$ have to do with the way the positive ionic charge is distributed between adjacent $\mathrm{Ir}^{4+} \mathrm{O}_{2}$ and $\mathrm{A}^{2+} \mathrm{O}$ layers, having in contrast to the 214 cuprate superconductors, for example, more positive charge in the TM-O planes. This almost compensates the 'local' tetragonal field arising from the $z$ axis elongation of the $\mathrm{IrO}_{6}$ octahedra in $\mathrm{Ba}_{2} \mathrm{IrO}_{4}$ and overcompensates it in $\mathrm{Sr}_{2} \mathrm{IrO}_{4}$.

The subtle interplay between local distortions of the $\mathrm{O}$ ligand cage and additional uniaxial fields associated with the anisotropic extended surroundings opens new perspectives on $\operatorname{strain}^{44}$ and pressure $^{19}$ experiments in square-lattice iridates, for example, in connection to the spin-flop transition earlier predicted in $\mathrm{Sr}_{2} \mathrm{IrO}_{4}$ (refs 12,36). It also opens up the perspective of manipulating this way the $d$-level ordering in oxide heterostructures with highly charged, trivalent and tetravalent species. Compounds with tetravalent species within the $\mathrm{TM}-\mathrm{O}_{2}$ layers, in particular, given the nonpolar character of the quasi $2 \mathrm{D}$ sheets, provide ideal playgrounds to explore the mechanism of $d$-level ordering pointed out here since that will not be hindered by 'interface' charge redistribution and structural reconstruction occuring in polar heterostructures from polar discontinuities ${ }^{50,51}$. A recent experimental realization of such mixed, tetravalent/ divalent TM-oxide interfaces is for example the $\mathrm{SrRuO}_{3} / \mathrm{NiO}$ interface ${ }^{52}$, one system that requires in this respect closer theoretical examination.

\section{Methods}

Single-site magnetic properties. The $g$ factors were obtained by computations on clusters which contain one central $\mathrm{IrO}_{6}$ octahedron, the four $\mathrm{NN} \mathrm{IrO}_{6}$ octahedra and the nearby ten $\mathrm{Sr} / \mathrm{Ba}$ ions. The solid-state surroundings were modelled as a large array of point charges fitted to reproduce the crystal Madelung field in the cluster region. To obtain a clear picture on crystal-field effects and spinorbit interactions at the central Ir site, we cutoff the magnetic couplings with the adjacent Ir ions by replacing the tetravalent open-shell $d^{5}$ NNs with tetravalent closed-shell $\mathrm{Pt}^{4+} t_{2 \mathrm{~g}}^{6}$ species. This is a usual procedure in quantum chemistry studies on TM systems, see for example, refs 42,45,53-56. We used energyconsistent relativistic pseudopotentials and valence basis sets of quadruple-zeta quality supplemented with $f$ polarization functions for the central $\operatorname{Ir}_{\text {ion }}{ }^{57}$ and all-electron triple-zeta basis sets for the six adjacent ligands ${ }^{58}$. For the TM NNs, we applied energy-consistent relativistic pseudopotentials and triple-zeta basis functions ${ }^{57}$ along with minimal atomic-natural-orbital basis sets ${ }^{59}$ for the Os coordinating those TM sites but not shared with the central octahedron. The Sr and $\mathrm{Ba}$ species were modelled by divalent total-ion effective potentials supplemented with a single $s$ function ${ }^{60}$. All O $2 p$ and metal $t_{2 \mathrm{~g}}$ electrons at the central octahedron were correlated in the MRCI calculations. The latter are performed with single and double substitutions with respect to the CASSCF reference (for technicalities, see refs 61,62 ), which is referred to as MRCISD. To separate the metal $5 d$ and O $2 p$ valence orbitals into different groups, that is, central-octahedron and adjacent-octahedra orbitals, we used the Pipek-Mezey localization module ${ }^{63}$ available in MOLPRO. The computations with hypothetical $\left(Q_{\mathrm{TM}}-2 \Delta q\right)$ and $\left(Q_{\mathrm{A}}+\Delta q\right)$ ionic charges at the TM and $\mathrm{Sr} / \mathrm{Ba}$ sites next to the reference Ir ion were carried out as frozen-orbital multideterminant calculations (also referred to as CASCI) with three Ir $t_{2 \mathrm{~g}}$ and five electrons in the active space and orbitals optimized for $\Delta q=0$.

The spin-orbit treatment was performed according to the procedure described in ref. 30. In a first step, the scalar relativistic Hamiltonian is used to calculate correlated wavefunctions for a finite number of low-lying states, either at the CASSCF level or at the MRCI level. In a second step, the spin-orbit part is added to the initial scalar relativistic Hamiltonian, matrix elements of the aforementioned states are evaluated for this extended Hamiltonian and the resulting matrix is finally diagonalized to yield SO wavefunctions.

The $g$ factors were computed following the scheme proposed by Bolvin ${ }^{27}$ and Vancoillie et al. ${ }^{28}$ (for alternative formulations, see, for example, ref. 64). For the
KD GS $\{\psi, \bar{\psi}\}$, the Abragam-Bleaney tensor ${ }^{13,65}, \mathbf{G}=g g^{T}$ can be written in matrix form as

$$
\begin{aligned}
G_{k l} & =2 \sum_{u, v=\psi, \bar{\psi}}\left\langle u\left|\hat{L}_{k}+g_{e} \hat{S}_{k}\right| v\right\rangle\left\langle v\left|\hat{L}_{l}+g_{e} \hat{S}_{l}\right| u\right\rangle \\
& =\sum_{m=x, y, z}\left(\Lambda_{k m}+g_{e} \Sigma_{k m}\right)\left(\Lambda_{l m}+g_{e} \Sigma_{l m}\right),
\end{aligned}
$$

where

$$
\begin{array}{lll}
\Lambda_{k x}=2 \operatorname{Re}\left[\left\langle\bar{\psi}\left|\hat{L}_{k}\right| \psi\right\rangle\right], & \Sigma_{k x}=2 \operatorname{Re}\left[\left\langle\bar{\psi}\left|\hat{S}_{k}\right| \psi\right\rangle\right], \\
\Lambda_{k y}=2 \operatorname{Im}\left[\left\langle\bar{\psi}\left|\hat{L}_{k}\right| \psi\right\rangle\right], & \Sigma_{k y}=2 \operatorname{Im}\left[\left\langle\bar{\psi}\left|\hat{S}_{k}\right| \psi\right\rangle\right], \\
\Lambda_{k z}=2\left\langle\psi\left|\hat{L}_{k}\right| \psi\right\rangle, & \Sigma_{k z}=2\left\langle\psi\left|\hat{S}_{k}\right| \psi\right\rangle .
\end{array}
$$

The MEs of $\hat{L}$ are here provided by MOLPRO while those of $\hat{S}$ are derived using the conventional expressions for the generalized Pauli matrices :

$$
\begin{aligned}
\left(\hat{S}_{z}\right)_{M M^{\prime}} & =M \delta_{M M^{\prime}}, \\
\left(\hat{S}_{x}\right)_{M M^{\prime}} & =\frac{1}{2} \sqrt{(S+M)(S-M+1)} \delta_{M-1, M^{\prime}} \\
& +\frac{1}{2} \sqrt{(S-M)(S+M+1)} \delta_{M+1, M^{\prime}}, \\
\left(\hat{S}_{y}\right)_{M M^{\prime}} & =-\frac{i}{2} \sqrt{(S+M)(S-M+1)} \delta_{M-1, M^{\prime}} \\
& +\frac{i}{2} \sqrt{(S-M)(S+M+1)} \delta_{M+1, M^{\prime}} .
\end{aligned}
$$

$\mathbf{G}$ is next diagonalized and the $g$ factors are obtained as the possitive square roots of the three eigenvalues. The corresponding eigenvectors specify the rotation matrix to the main magnetic axes. In our case, the magnetic $z$ axis is along the crystallographic $c$ coordinate, while $x$ and $y$ are 'degenerate' and can be any two perpendicular directions in the $a b$ plane.

To cross-check the $g$-factor values computed with our subroutine, we further performed $g$-factor calculations using the module available within the ORCA quantum chemistry package ${ }^{66}$. We applied all-electron DKH (Douglas-Kroll-Hess) basis sets of triple-zeta quality for the TM ions ${ }^{67}$, triple-zeta basis functions for the ligands of the central octahedron ${ }^{58}$ and double-zeta basis functions for additional Os at the NN octahedra ${ }^{58}$. Dynamical correlation effects were accounted for by $N$ electron valence-state second-order perturbation theory (NEVPT2) ${ }^{68,69}$. CASSCF and NEVPT2 results are listed in Table 3, for both $\mathrm{Sr}_{2} \mathrm{IrO}_{4}$ and $\mathrm{Ba}_{2} \mathrm{IrO}_{4}$. It is seen that the data in Tables 1 and 3 compare very well and indicate the same overall trends.

Superexchange interactions in $\mathbf{S r}_{\mathbf{2}} \mathbf{I} \mathbf{r O}_{\mathbf{4}}$. NN magnetic coupling constants were obtained for $\mathrm{Sr}_{2} \mathrm{IrO}_{4}$ by calculations on an embedded cluster that includes two $\mathrm{IrO}_{6}$ octahedra as magnetically active units. As for the calculation of single-site magnetic properties, to accurately describe the charge distribution in the immediate neighbourhood, the adjacent $\mathrm{IrO}_{6}$ octahedra (six) and the closest $\mathrm{Sr}^{2}+$ ions (16) were also incorporated in the actual cluster. We used energy-consistent relativistic pseudopotentials along with quadruple-zeta basis sets for the valence shells of the two magnetically active Ir ions ${ }^{57}$, all-electron quintuple-zeta basis sets for the bridging ligand ${ }^{58}$ and triple-zeta basis functions for the other Os associated with the two reference octahedra ${ }^{58}$. We further employed polarization functions at the two central Ir sites and for the bridging anion, namely 2 Ir $f$ and $4 \mathrm{O} d$

functions ${ }^{57,58}$. Additional ions defining the NN octahedra, the nearby $\mathrm{Sr}^{2}+$ species and the farther crystalline surroundings were modelled as in the single-site study, see above.

\begin{tabular}{|c|c|c|c|}
\hline & $\delta_{t 2 \mathrm{~g}}(\mathrm{meV})$ & $\boldsymbol{g}_{\perp}$ & $g_{\|}$ \\
\hline \multicolumn{4}{|l|}{$\mathrm{Sr}_{2} \mathrm{IrO}_{4}:$} \\
\hline CASSCF & -127 & 1.66 & 2.23 \\
\hline NEVPT2 & -199 & 1.55 & 2.41 \\
\hline \multicolumn{4}{|l|}{$\mathrm{Ba}_{2} \mid \mathrm{rO}_{4}:$} \\
\hline CASSCF & 30 & 1.93 & 1.74 \\
\hline NEVPT2 & 70 & 2.01 & 1.58 \\
\hline
\end{tabular}

For two adjacent magnetic sites, the ${ }^{2} T_{2 \mathrm{~g}}\left(t_{2 \mathrm{~g}}^{5}\right) \otimes{ }^{2} T_{2 \mathrm{~g}}\left(t_{2 \mathrm{~g}}^{5}\right)$ manifold entails nine singlet and nine triplet states. The CASSCF optimization was carried out for an average of these nine singlet and nine triplet eigenfunctions of the scalar relativistic Hamiltonian $\mathcal{H}_{\mathrm{SR}}^{i, j}$. In the subsequent MRCI treatment, only the $\operatorname{Ir} t_{2 \mathrm{~g}}$ and the $\mathrm{O} 2 p$ electrons at the bridging ligand site were correlated. Results in good agreement with the experimental data were recently obtained with this computational approach for related $5 d^{5}$ iridates ${ }^{21,70}$.

Diagonalization of the Hamiltonian $\mathcal{H}_{\mathrm{SR}}^{i, j}+\mathcal{H}_{\mathrm{SO}}^{i, j}$ in the basis of the lowest nine singlet and nine triplet states provides a total of 36 spin-orbit-coupled $\left|j_{\text {eff }}^{i}, j_{\text {eff }}^{j}\right\rangle$

Results as obtained with the ORCA program ${ }^{66}$ are shown. Only the ${ }^{2} T_{2 g}\left(t_{2 g}^{5}\right)$ states were included in the CASSCF optimization and in the spin-orbit treatment. 
eigenfunctions, namely, four $|1 / 2,1 / 2\rangle$, eight $|1 / 2,3 / 2\rangle$, eight $|3 / 2,1 / 2\rangle$ and sixteen $|3 / 2,3 / 2\rangle$ states. In the simplest picture, the lowest four $|1 / 2,1 / 2\rangle$ roots imply either singlet or triplet coupling of the spin-orbit $j_{\text {eff }}=1 / 2$ (or, more generally, pseudospin $\tilde{S}=1 / 2$ ) on-site objects and are separated from higher-lying states by a gap of $\gtrsim 0.5 \mathrm{eV}$, much larger than the strength of the intersite exchange. It is this set of lowest four spin-orbit MRCI roots that we map onto the eigenstates of the effective two-site (pseudo)spin Hamiltonian (1). The Zeeman interaction shows up on the quantum chemistry side as $\mathcal{H}_{\mathrm{Z}}^{i, j}=\mu_{\mathrm{B}}\left(\hat{\mathbf{L}}^{, i, j}+g_{e} \hat{\mathbf{S}}^{i, j}\right) \cdot \mathbf{h}$, where $\hat{\mathbf{L}}^{i, j}$ and $\hat{\mathbf{S}}^{i, j}$ are transformed to the spin-orbit-coupled basis using the spin-orbit wavefunctions as unitary transformation matrix. MEs of the $a b$ initio model Hamiltonian $\mathcal{H}_{\mathrm{SR}}^{i, j}+\mathcal{H}_{\mathrm{SO}}^{i, j}+\mathcal{H}_{\mathrm{Z}}^{i, j}$ are shown in Table 4 . Diagonal components show the energies of the zero-field states, while the off-diagonal MEs describe the coupling to magnetic field.

For the experimentally determined crystal structure of $\mathrm{Sr}_{2} \mathrm{IrO}_{4}$ (ref. 23), the two-octahedra $\left[\mathrm{Ir}_{2} \mathrm{O}_{11}\right]$ cluster displays $C_{2 v}$ symmetry. Having the $x$ axis along the $\langle 110\rangle$ crystallographic direction ${ }^{23}$ and $z \| c$, the effective anisotropic couplings read $\mathbf{D}=(0,0, D)$

$$
\overline{\bar{\Gamma}}^{(x)}=\left(\begin{array}{ccc}
\Gamma_{x x} & 0 & 0 \\
0 & \Gamma_{y y} & 0 \\
0 & 0 & \Gamma_{z z}
\end{array}\right)
$$

for Ir-Ir links along $x$ and

$$
\overline{\bar{\Gamma}}^{(y)}=\left(\begin{array}{ccc}
\Gamma_{y y} & 0 & 0 \\
0 & \Gamma_{x x} & 0 \\
0 & 0 & \Gamma_{z z}
\end{array}\right)
$$

for Ir-Ir links along $y$, with $\Gamma_{x x}+\Gamma_{y y}+\Gamma_{z z}=0$. The uniform and staggered components of the $\overline{\mathbf{g}}$ tensor take for individual Ir sites the following form :

$$
\overline{\mathbf{g}}_{i}=\overline{\mathbf{g}}_{i}^{\mathrm{u}} \pm \overline{\mathbf{g}}_{i}^{\mathrm{s}}=\left(\begin{array}{ccc}
g_{x x} & 0 & 0 \\
0 & g_{y y} & 0 \\
0 & 0 & g_{z z}
\end{array}\right) \pm\left(\begin{array}{ccc}
0 & g_{x y} & 0 \\
g_{x y} & 0 & 0 \\
0 & 0 & 0
\end{array}\right) .
$$

$g_{z z}=g_{\|}$while $g_{x x}$ and $g_{y y}$ are directly related to $g_{\perp}$ but not restricted to be equal due to the lower symmetry of the two-octahedra cluster as compared with the $\mathrm{IrO}_{6}$ unit.

To solve now the actual problem, we need to transform the effective spin Hamiltonian (1) to the same form as the ab initio Hamiltonian shown in Table 4, that is, diagonal in zero magnetic field. The result of such a transformation is shown in Table 5. Direct correspondence between homologous MEs in the two arrays yields a set of eight independent equations that finally allow to derive hard values for all effective coupling constants that enter expression (1). While the

\section{Table 4 | Matrix elements of the $a b$ initio spin-orbit Hamiltonian.}

\begin{tabular}{ccccc}
$\mathcal{H}^{i, j}$ & $|\tilde{\boldsymbol{s}}\rangle$ & $\left|\boldsymbol{t}_{\mathbf{x}}\right\rangle$ & $\left|\boldsymbol{t}_{\mathbf{y}}\right\rangle$ & $\left|\tilde{\mathbf{t}}_{\mathbf{z}}\right\rangle$ \\
\hline$\langle\tilde{s}|$ & 0 & $0.2308 i \mu_{\mathrm{B}} h_{y}$ & $-0.1768 i \mu_{\mathrm{B}} h_{x}$ & 0 \\
$\left\langle t_{x}\right|$ & $-0.2308 i \mu_{\mathrm{B}} h_{y}$ & 48.3328 & $2.3083 i \mu_{\mathrm{B}} h_{z}$ & $-1.6854 i \mu_{\mathrm{B}} h_{\mathrm{y}}$ \\
$\left\langle t_{y}\right|$ & $0.1768 i \mu_{\mathrm{B}} h_{x}$ & $-2.3083 i \mu_{\mathrm{B}} h_{z}$ & 48.9626 & $1.6266 i \mu_{\mathrm{B}} h_{x}$ \\
$\left\langle\tilde{t}_{z}\right|$ & 0 & $1.6854 i \mu_{\mathrm{B}} h_{y}$ & $-1.6266 i \mu_{\mathrm{B}} h_{x}$ & 49.0630 \\
\hline
\end{tabular}

The latter is described by expression (6). Results of spin-orbit MRCl calculations are shown $(\mathrm{meV})$. The two-site singlet and (split) triplet states are labelled $|\tilde{s}\rangle$ and $\left\{\left|t_{x}\right\rangle,\left|t_{y}\right\rangle,\left|\tilde{t}_{z}\right\rangle\right\}$, respectively. Due to the antisymmetric exchange, $|\tilde{s}\rangle$ and $\left|\tilde{t}_{z}\right\rangle$ are admixtures of 'pure' $|0,0\rangle$ and $|1,0\rangle$ spin functions. results for the intersite exchange interactions in (1) and $(10,11)$ are shown in Table 2, the $\overline{\overline{\mathbf{g}}}$-tensor data obtained from the two-octahedra calculations are $g_{x x}=1.64, g_{y y}=1.70, g_{z z}=2.31$ and $g_{x y}=0.02$. The way the additional parameters introduced in Table 5 are defined is explained in Table 6 . The intermediate steps followed to arrive to the matrix form provided in Table 5 are outlined in Supplementary Tables I-III and the Supplementary Methods.

Spin-wave calculations. For understanding all details of the ESR spectrum, we carried out a spin-wave analysis using the Hamiltonian (1) and a site-factorized variational GS wave function

$$
|\Psi\rangle=\prod_{\text {u.c. }}\left|\psi_{A}\right\rangle\left|\psi_{B}\right\rangle
$$

where for each sublattice index $L \in\{A, B\}$,

$$
\left|\psi_{L}\right\rangle=\cos \frac{\alpha_{L}}{2}|\uparrow\rangle+e^{i \phi_{L}} \sin \frac{\alpha_{L}}{2}|\downarrow\rangle .
$$

For the $2 \mathrm{D}$ unit cell displayed in Fig. $3 \mathrm{a}, \mathrm{D}_{2 d}$ symmetry is considered.

The spin components of the GS configuration depend on the $\alpha_{L}$ and $\phi_{L}$ variational parameters as

$$
\begin{gathered}
S_{L}^{x}=\frac{1}{2} \sin \alpha_{L} \cos \phi_{L}, \\
S_{L}^{y}=\frac{1}{2} \sin \alpha_{L} \sin \phi_{L}, \\
S_{L}^{z}=\frac{1}{2} \cos \alpha_{L} .
\end{gathered}
$$

For magnetic fields parallel to the $c$ axis, the GS energy only depends on the parameters $\alpha_{A}=\alpha_{B}=\alpha$ and $\phi_{A}-\phi_{B}=\delta \phi . \delta \phi$ is the angle between neighbouring in-plane spins and $\alpha$ describes how much the spins are tilted away from the $c$ axis. For $\alpha=\pi / 2$ the spins are lying within the $a b$ plane, while $\alpha=0$ corresponds to the fully polarized high-magnetic-field case. The GS energy

$$
\begin{aligned}
E_{0} & =\left(J+\Gamma_{z z}\right) \cos \alpha^{2}-g_{\|} h_{\|} \cos \alpha \\
& +\left(\left(J-\frac{1}{2} \Gamma_{z z}\right) \cos \delta \phi-D \sin \delta \phi\right) \sin \alpha^{2}
\end{aligned}
$$

is minimized when

$$
\begin{gathered}
\delta \phi=-\arccos \frac{2 J-\Gamma_{z z}}{\tilde{J}}, \\
\alpha=\arccos \frac{g_{\|} h_{\|}}{2 J+2 \Gamma_{z z}+\tilde{J}} .
\end{gathered}
$$

For zero field we find that $\alpha=0$, the spins are confined to the $a b$ plane (that is, to the $\mathrm{IrO}_{2}$ layer) and the angle $\delta \phi$ is controlled by the strength of $D . \delta \phi$ is not affected by fields along the $c$ axis while $\alpha$ changes smoothly from $\pi / 2$ to 0 with increasing the field strength.

The two magnons characteristic for spin-1/2 antiferromagnets are related to states orthogonal to $\left|\psi_{A}\right\rangle$ and $\left|\psi_{B}\right\rangle$. The $4 \times 4$ Hamiltonian defining these magnons can be derived by using the well-known Holstein-Primakoff approach and diagonalized through a Bogoliubov transformation. For $h \| c$, the two spin-wave modes are the gapless $v_{\| 1}=0$ Goldstone mode, corresponding to $\mathrm{U}(1)$ symmetry breaking, and the gapped mode given by (2). When the magnetic field lies in the $a b$ plane, the $z$ component of Ir spins remains zero (with $\alpha=\pi / 2$ ) and the GS energy

Table 5 I Matrix elements of the effective spin Hamiltonian.

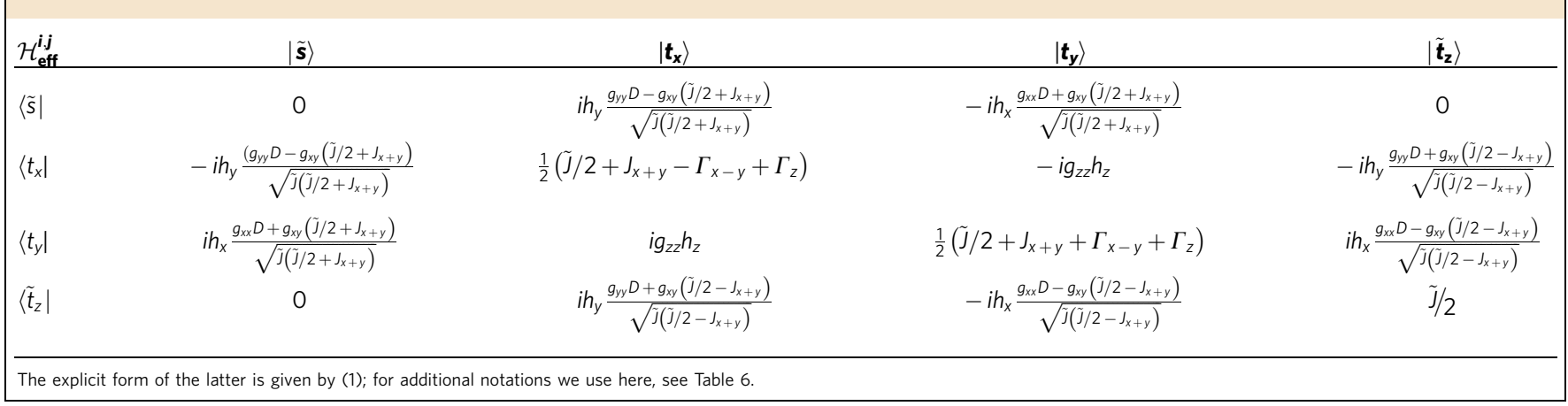




\section{Table 6 | Notations used for anisotropic exchange coupling} parameters in $\mathrm{Sr}_{2} \mathrm{IrO}_{4}$.

\begin{tabular}{|c|c|c|c|}
\hline & $\begin{array}{c}\boldsymbol{J}_{\boldsymbol{t}} \boldsymbol{\Gamma}_{x \mathrm{x}}=-\Gamma_{y y}-\Gamma_{z z} \\
\text { (present study) }\end{array}$ & $\begin{array}{r}J_{x}, J_{y}, J_{z} \\
\text { (ref. 36) }\end{array}$ & $\begin{array}{c}J_{,} \delta J_{x y}, \delta J_{z}(x \text { bond) } \\
\text { (refs } 12,35,36)\end{array}$ \\
\hline$J_{x+y}$ & $J-\Gamma_{z z} / 2$ & $\left(J_{x}+J_{y}\right) / 2$ & $J+\delta J_{x y} / 2$ \\
\hline$\tilde{\jmath}$ & $2 \sqrt{D^{2}+J_{x+y}^{2}}$ & $2 \sqrt{D^{2}+J_{x+y}^{2}}$ & $2 \sqrt{D^{2}+J_{x+y}^{2}}$ \\
\hline$\Gamma_{x-y}$ & $\left(\Gamma_{x x}-\Gamma_{y y}\right) / 2$ & $\left(J_{x}-J_{y}\right) / 2$ & $\left\{\begin{array}{cl}\delta J_{x y} & \text { for }\left\langle t_{x}|\mathcal{H}| t_{x}\right\rangle \\
0 & \text { for }\left\langle t_{y}|\mathcal{H}| t_{y}\right\rangle\end{array}\right.$ \\
\hline$\Gamma_{z}$ & $3 / 2 \Gamma_{z z}$ & $J_{z}-J_{x+y}$ & $\delta J_{z}$ \\
\hline
\end{tabular}

The definitions on the left-hand side are applied in Table 5. Other conventions presently employed in the literature are also shown for comparison.

only depends on the $\delta \phi$ angle :

$$
E_{0}=\left(J+\frac{\Gamma_{z z}}{2}\right) \cos \delta \phi-g_{x} h_{x} \cos \frac{\delta \phi}{2}-D \sin \delta \phi
$$

For simplicity, we select $x$ for the direction of the magnetic field. The result is, however, independent of how this choice is made as there is no anisotropy within the $a b$ plane.

An infinitesimally small in-plane field fixes the direction of uniform magnetization as $m=-\cos \frac{\delta \phi}{2}$. At finite field both modes thus become gapped. To determine the uniform magnetization $m$ one needs to minimize equation (21), which to leading order in magnetic field leads to equation (5). The first term of equation (5) corresponds to the zero-field moment, which arises due to the canting induced by the DM interaction. This ferromagnetic order-parameter is further enhanced in finite $h_{\perp}$ field. As long as $h_{\perp}$ is small equation (5) remains valid.

As discussed in the main text, in low fields, $m$ can be approximated by its field independent value ${ }^{26}$. Using the quantum chemically derived interaction parameters (see Table 2), we then find $m \approx 0.12 \mu_{\mathrm{B}}$, in good agreement with recent experiments $s^{8,40}$. Yet the zero-field gap comes out too large as compared to experiment. A good fit can nevertheless be reached with $J, D$ and $g$ values as obtained in the MRCI treatment and by increasing $\Gamma_{z z}$ from 0.42 to $0.98 \mathrm{meV}$.

\section{References}

1. Heber, J. Materials science: Enter the oxides. Nature 459, 28 (2009).

2. Mannhart, J. \& Schlom, D. G. Oxide interfaces - an opportunity for electronics. Science 327, 1607 (2010).

3. Chakhalian, J. et al. Orbital reconstruction and covalent bonding at an oxide interface. Science 318, 1114 (2007).

4. Benckiser, E. et al. Orbital reflectometry of oxide heterostructures. Nat. Mater. 10, 189 (2011).

5. Hwang, H. Y. et al. Emergent phenomena at oxide interfaces. Nat. Mater. 11, 103 (2012).

6. Leggett, J. What DO we know about high $\mathrm{T}_{\mathrm{c}}$ ? Nat. Phys. 2, 134-136 (2006).

7. Kim, J. et al. Novel Jeff $=1 / 2$ Mott state induced by relativistic spin-orbit coupling in $\mathrm{Sr}_{2} \mathrm{IrO}_{4}$. Phys. Rev. Lett. 101, 076402 (2008).

8. Kim, J. et al. Phase-sensitive observation of a spin-orbital Mott state in $\mathrm{Sr}_{2} \mathrm{IrO}_{4}$. Science 323, 1329 (2009).

9. Kim, J. et al. Magnetic excitation spectra of $\mathrm{Sr}_{2} \mathrm{IrO}_{4}$ probed by resonant inelastic X-ray scattering: Establishing links to cuprate superconductors. Phys. Rev. Lett. 108, 177003 (2012).

10. Kim, Y. K. et al. Fermi arcs in a doped pseudospin-1/2 Heisenberg antiferromagnet. Science 345, 187 (2014).

11. Kim, J. et al. Excitonic quasiparticles in a spin-orbit Mott insulator. Nat. Commun. 5, 4453 (2014).

12. Jackeli, G. \& Khaliullin, G. Mott insulators in the strong spin-orbit coupling limit: From Heisenberg to a quantum compass and Kitaev models. Phys. Rev. Lett. 102, 017205 (2009).

13. Abragam, A. \& Bleaney, B. Electron Paramagnetic Resonance of Transition Ions (Clarendon Press, 1970).

14. Boseggia, S. et al. Robustness of basal-plane antiferromagnetic order and the $\mathrm{J}_{\text {eff }}=1 / 2$ state in single-layer iridate spin-orbit Mott insulators. Phys. Rev. Lett. 110, 117207 (2013).

15. Thornley, J. H. M. The magnetic properties of $\left(\mathrm{IrX}_{6}\right)^{2-}$ complexes. J. Phys. C 1, 1024 (1968).

16. Hill, N. J. Electron paramagnetic resonance of osmium-doped trichlorotris (diethylphenylphosphine) rhodium (III). J. Chem. Soc. Faraday Trans. 2 68, 427-434 (1972).
17. Andlauer, B., Schneider, J. \& Tolksdorf, W. Optical absorption, fluorescence, and electron spin resonance of $\mathrm{Ir}^{4+}$ on octahedral sites in $\mathrm{Y}_{3} \mathrm{Ga}_{5} \mathrm{O}_{12}$. Phys. Stat. Sol. B 73, 533 (1976).

18. Stebler, A., Ammeter, J. M., Furholz, U. \& Ludi, A. Electronic properties of hexaaquaruthenium (III): EPR and optical spectra of $\mathrm{Ru}\left(\mathrm{H}_{2} \mathrm{O}\right)_{6}^{3}+$ in alum crystals. Inorg. Chem. 23, 2764 (1984).

19. Haskel, D. et al. Pressure tuning of the spin-orbit coupled ground state in $\mathrm{Sr}_{2} \mathrm{IrO}_{4}$. Phys. Rev. Lett. 109, 027204 (2012).

20. Liu, X. et al. Testing the validity of the strong spin-orbit-coupling limit for octahedrally coordinated iridate compounds in a model system $\mathrm{Sr}_{3} \mathrm{CuIrO}_{6}$. Phys. Rev. Lett. 109, 157401 (2012).

21. Bogdanov, N. A., Katukuri, V. M., Stoll, H., van den Brink, J. \& Hozoi, L. Post-perovskite $\mathrm{CaIrO}_{3}: \mathrm{A} \mathrm{j}=1 / 2$ quasi-one-dimensional antiferromagnet. Phys. Rev. B 85, 235147 (2012).

22. Hozoi, L. et al. Longer-range lattice anisotropy strongly competing with spin-orbit interactions in pyrochlore iridates. Phys. Rev. B 89, 115111 (2014).

23. Crawford, M. K. et al. Structural and magnetic studies of $\mathrm{Sr}_{2} \mathrm{IrO}_{4}$. Phys. Rev. B 49, 9198 (1994).

24. Ballhausen, C. J. Introduction to Ligand Field Theory (McGraw-Hill, 1962).

25. Oshikawa, M. \& Affleck, I. Field-induced gap in $S=1 / 2$ antiferromagnetic chains. Phys. Rev. Lett. 79, 2883 (1997).

26. Bahr, S. et al. Low-energy magnetic excitations in the spin-orbital Mott insulator $\mathrm{Sr}_{2} \mathrm{IrO}_{4}$. Phys. Rev. B 89, 180401 (2014).

27. Bolvin, H. An alternative approach to the g-matrix: Theory and applications. Chem. Phys. Chem. 7, 1575 (2006).

28. Vancoillie, S., Malmqvist, P. A. \& Pierloot, K. Calculation of EPR g tensors for transition-metal complexes based on multiconfigurational perturbation theory (CASPT2). Chem. Phys. Chem. 8, 1803 (2007).

29. Helgaker, T., Jørgensen, P. \& Olsen, J. Molecular Electronic-Structure Theory (Wiley, 2000).

30. Berning, M. Schweizer, Werner, H.-J., Knowles, P. J. \& Palmieri, P. Spin-orbit matrix elements for internally contracted multireference configuration interaction wave-functions. Mol. Phys. 98, 1823-1833 (2000).

31. Werner, H.-J., Knowles, P. J., Knizia, G., Manby, F. R. \& Schutz, M. MOLPRO 2012. (University of Cardiff, see http://www.molpro.net).

32. Katukuri, V. M. et al. Electronic structure of low-dimensional $4 \mathrm{~d}^{5}$ oxides: interplay of ligand distortions, overall lattice anisotropy, and spin-orbit interactions. Inorg. Chem. 53, 4833 (2014).

33. Okabe, $\mathrm{H}$. et al. $\mathrm{Ba}_{2} \mathrm{IrO}_{4}$ : A spin-orbit Mott insulating quasi-two-dimensional antiferromagnet. Phys. Rev. B 83, 155118 (2011).

34. Subramanian, M. A. et al. $\mathrm{Sr}_{2} \mathrm{RhO}_{4}$ and $\mathrm{Sr}_{2} \mathrm{IrO}_{4}$ : Structural and magnetic studies of $4 \mathrm{~d}$ and $5 \mathrm{~d}$ transition metal analogs of $\mathrm{La}_{2} \mathrm{CuO}_{4}$. Phys. C 235-240, 743 (1994).

35. Kim, B. H., Khaliullin, G. \& Min, B. I. Magnetic couplings, optical spectra, and spin-orbit exciton in 5d electron Mott insulator $\mathrm{Sr}_{2} \mathrm{IrO}_{4}$. Phys. Rev. Lett. 109, 167205 (2012).

36. Perkins, N. B., Sizyuk, Y. \& Wolfle, P. Interplay of many-body and singleparticle interactions in iridates and rhodates. Phys. Rev. B 89, 035143 (2014).

37. Boseggia, S. et al. Locking of iridium magnetic moments to the correlated rotation of oxygen octahedra in $\mathrm{Sr}_{2} \mathrm{IrO}_{4}$ revealed by X-ray resonant scattering. J. Phys.: Condens. Matter 25, 422202 (2013).

38. Ye, F. et al. Magnetic and crystal structures of $\mathrm{Sr}_{2} \mathrm{IrO}_{4}$ : A neutron diffraction study. Phys. Rev. B 87, 140406(R) (2013).

39. Katukuri, V. M. et al. Mechanism of basal-plane antiferromagnetism in the spin-orbit driven iridate $\mathrm{Ba}_{2} \mathrm{IrO}_{4}$. Phys. Rev. X 4, 021051 (2014).

40. Cao, G., Bolivar, J., McCall, S., Crow, J. E. \& Guertin, R. P. Weak ferromagnetism, metal-to-nonmetal transition, and negative differential resistivity in single-crystal $\mathrm{Sr}_{2} \mathrm{IrO}_{4}$. Phys. Rev. B 57, 11039(R) (1998).

41. Kim, B. J. et al. Missing xy-band Fermi surface in $4 \mathrm{~d}$ transition-metal oxide $\mathrm{Sr}_{2} \mathrm{RhO}_{4}$ : Effect of the octahedra rotation on the electronic structure. Phys. Rev. Lett. 97, 106401 (2006).

42. Bogdanov, N. A., Maurice, R., Rousochatzakis, I., van den Brink, J. \& Hozoi, L. Magnetic state of pyrochlore $\mathrm{Cd}_{2} \mathrm{Os}_{2} \mathrm{O}_{7}$ emerging from strong competition of ligand distortions and longer-range crystalline anisotropy. Phys. Rev. Lett. 110, 127206 (2013).

43. Fang, Z., Nagaosa, N. \& Terakura, K. Orbital-dependent phase control in $\mathrm{Ca}_{2}-{ }_{x} \mathrm{Sr}_{x} \mathrm{RuO}_{4}(0<\mathrm{x}<0.5)$. Phys. Rev. B 69, 045116 (2004).

44. Lupascu, A. et al. Tuning magnetic coupling in $\mathrm{Sr}_{2} \mathrm{IrO}_{4}$ thin films with epitaxial strain. Phys. Rev. Lett. 112, 147201 (2014).

45. Hozoi, L., Siurakshina, L., Fulde, P. \& van den Brink, J. Ab initio determination of $\mathrm{Cu} 3 \mathrm{~d}$ orbital energies in layered copper oxides. Sci. Rep. 1, 65 (2011).

46. $\mathrm{Wu}, \mathrm{H}$. et al. Orbital order in $\mathrm{La}_{0.5} \mathrm{Sr}_{1.5} \mathrm{MnO}_{4}$ : Beyond a common local JahnTeller picture. Phys. Rev. B 84, 155126 (2011).

47. Braden, M., Andre, G., Nakatsuji, S. \& Maeno, Y. Crystal and magnetic structure of $\mathrm{Ca}_{2} \mathrm{RuO}_{4}$ : Magnetoelastic coupling and the metal-insulator transition. Phys. Rev. B 58, 847 (1998).

48. Fujiyama, S. et al. Spin and orbital contributions to magnetically ordered moments in 5d layered perovskite $\mathrm{Sr}_{2} \mathrm{IrO}_{4}$. Phys. Rev. Lett. 112, 016405 (2014). 
49. Bolvin, $\mathrm{H}$. Theoretical determination of the excited states and of g-factors of the Creutz-Taube ion, $\left[\left(\mathrm{NH}_{3}\right)_{5}-\mathrm{Ru}-\text { pyrazine-Ru-(NH3)5 }\right]^{5+}$. Inorg. Chem. 46, 417 (2007).

50. Ohtomo, A. \& Hwang, H. Y. A high-mobility electron gas at the $\mathrm{LaAlO}_{3} / \mathrm{SrTiO}_{3}$ heterointerface. Nature 427, 423 (2004).

51. Koster, G., Brinkman, A., Hilgenkamp, H., Rijnders, A. J. H. M. \& Blank, D. H. A. High- Tc superconducting thin films with composition control on a sub-unit cell level; the effect of the polar nature of the cuprates. J. Phys.: Condens. Matter 20, 264007 (2008).

52. Ning, X., Wang, Z. \& Zhang, Z. Exchange bias effect and large coercivity enhancement in $\mathrm{SrRuO}_{3} / \mathrm{NiO}$ multilayers. J. Phys. D: Appl. Phys. 46, 452001 (2013).

53. de Graaf, C., Sousa, C. \& Broer, R. Ionization and excitation energies in $\mathrm{CuCl}$ and $\mathrm{NiO}$ within different embedding schemes. J. Mol. Struct. 458, 53 (1999).

54. Hozoi, L. et al. Theoretical characterization of the ground and optically excited states of $\alpha^{\prime}-\mathrm{NaV}_{2} \mathrm{O}_{5}$. Phys. Rev. Lett. 89, 076407 (2002).

55. Domingo, A., Rodrlguez-Fortea, A., Swart, M., de Graaf, C. \& Broer, R. Ab initio absorption spectrum of $\mathrm{NiO}$ combining molecular dynamics with the embedded cluster approach in a discrete reaction field. Phys. Rev. B 85, 155143 (2012).

56. Maurice, R. et al. Single-ion magnetic anisotropy and isotropic magnetic couplings in the metal-organic framework $\mathrm{Fe}_{2}$ (dobdc). Inorg. Chem. 52, 9379 (2013).

57. Figgen, D., Peterson, K. A., Dolg, M. \& Stoll, H. Energy-consistent pseudopotentials and correlation consistent basis sets for the $5 \mathrm{~d}$ elements Hf-Pt. J. Chem. Phys. 130, 164108 (2009).

58. Dunning, T. H. Gaussian basis sets for use in correlated molecular calculations. I. The atoms boron through neon and hydrogen. J. Chem. Phys. 90, 1007 (1989).

59. Pierloot, K., Dumez, B., Widmark, P.-O. \& Roos, B. Density matrix averaged atomic natural orbital (ANO) basis sets for correlated molecular wave functions. Theor. Chim. Acta 90, 87 (1995).

60. Fuentealba, P., von Szentpaly, L., Preuss, H. \& Stoll, H. Pseudopotential calculations for alkaline-earth atoms. J. Phys. B 18, 1287 (1985)

61. Werner, H.-J. \& Knowles, P. J. An efficient internally contracted multiconfiguration-reference configuration interaction method. J. Chem. Phys. 89, 5803 (1988).

62. Knowles, P. J. \& Werner, H.-J. Internally contracted multiconfigurationreference configuration interaction calculations for excited states. Theor. Chim. Acta 84, 95 (1992).

63. Pipek, J. \& Mezey, P. G. A fast intrinsic localization procedure applicable for ab initio and semiempirical linear combination of atomic orbital wave functions. J. Chem. Phys. 90, 4916 (1989).

64. Chibotaru, L. F. \& Ungur, L. Ab initio calculation of anisotropic magnetic properties of complexes. I. Unique definition of pseudospin Hamiltonians and their derivation. J. Chem. Phys. 137, 064112 (2012).

65. Gerloch, M. \& McMeeking, R. F. Paramagnetic properties of unsymmetrical transition-metal complexes. J. Chem. Soc., Dalton Trans. 2443 (1975).

66. Neese, F. The ORCA program system. Wiley Interdiscip. Rev. Comput. Mol. Sci. 2, 73-78 (2012)
67. Pantazis, D. A., Chen, X. Y., Landis, C. R. \& Neese, F. All-electron scalar relativistic basis sets for third-row transition metal atoms. J. Chem. Theory Comput. 4, 908 (2008).

68. Angeli, C., Cimiraglia, R. \& Malrieu, J.-P. N-electron valence state perturbation theory: a fast implementation of the strongly contracted variant. Chem. Phys. Lett. 350, 297 (2001).

69. Schapiro, I., Sivalingam, K. \& Neese, F. Assessment of n-electron valence state perturbation theory for vertical excitation energies. J. Chem. Theory Comput. 9, 3567 (2013).

70. Katukuri, V. M. et al. Kitaev interactions between $j=1 / 2$ moments in honeycomb $\mathrm{Na}_{2} \mathrm{IrO}_{3}$ are large and ferromagnetic: insights from ab initio quantum chemistry calculations. New J. Phys. 16, 013056 (2014).

\section{Acknowledgements}

We thank G. Jackeli, G. Khaliullin, P. Fulde, and H. Takagi for fruitful discussions. N.A.B. and L.H. acknowledge financial support from the Erasmus Mundus Programme of the European Union and the German Research Foundation (Deutsche Forschungsgemeinschaft, DFG), respectively. Experimental work was in part supported through the DFG project KA 1694/8-1.

\section{Author contributions}

V.K. conducted the ESR experiments. N.A.B. and V.M.K. carried out the $a b$ initio quantum chemistry calculations and subsequent mapping of the $a b$ initio data onto the effective spin Hamiltonian, with assistance from L.H., V.Y., J.R. and V.K. J.R. performed the spin-wave analysis. L.H., V.K., J.v.d.B. and B.B. designed the project. L.H., J.v.d.B., V.K., N.A.B., V.M.K. and J.R. wrote the paper, with contributions from all other coauthors.

\section{Additional information}

Supplementary Information accompanies this paper at http://www.nature.com/ naturecommunications

Competing financial interests: The authors declare no competing financial interests.

Reprints and permission information is available online at http://npg.nature.com/ reprintsandpermissions/

How to cite this article: Bogdanov, N. A. et al. Orbital reconstruction in nonpolar tetravalent transition-metal oxide layers. Nat. Commun. 6:7306 doi: $10.1038 /$ ncomms8306 (2015).

cc) (i) This work is licensed under a Creative Commons Attribution 4.0 cc. International License. The images or other third party material in this article are included in the article's Creative Commons license, unless indicated otherwise in the credit line; if the material is not included under the Creative Commons license, users will need to obtain permission from the license holder to reproduce the material. To view a copy of this license, visit http://creativecommons.org/licenses/by/4.0/ 